\title{
Postpancreatoduodenectomy Hemorrhage
} Treated by Combined Transcatheter Arterial Embolization and Superior Mesenteric Artery to Iliac Artery Bypass: Report of a Case

Hayato Sasaki, Yoshiaki Murakami, Kenichiro Uemura, Takeshi Sudo, Yasushi Hashimoto, Naru Kondo, Taijiro Sueda

Department of Surgery, Institute of Biomedical and Health Sciences, Hiroshima University, Hiroshima, Japan

\begin{abstract}
Postpancreatectomy hemorrhage is a potentially life-threatening complication. We report herein our experience with a 65-year-old man with locally advanced pancreatic adenocarcinoma who underwent pancreatoduodenectomy with lymphadenectomy following neoadjuvant chemoradiotherapy. On postoperative day 45 , he developed massive hematemesis. Angiography revealed active bleeding from the common hepatic artery, and transcatheter coil embolization of that vessel was successfully performed. On postoperative day 64, he again developed massive hematemesis. Angiography revealed active bleeding from the proximal superior mesenteric artery. Immediately after coil embolization of that vessel, bypass grafting between the superior mesenteric artery and the right common iliac artery was performed, using a greater saphenous vein graft. The combination of embolization and bypass grafting is an option for treatment of bleeding from the superior mesenteric artery in an emergent situation.
\end{abstract}

Key words: Superior mesenteric artery - Bleeding - Bypass - Pancreatoduodenectomy Postpancreatectomy hemorrhage

$\mathrm{P}$ ostpancreatectomy hemorrhage (PPH) is a rare but life-threatening complication, often associated with the presence of a pancreatic fistula or intraabdominal abscess. ${ }^{1}$ The mortality associated with arterial bleeding after pancreatoduodenectomy is reportedly between $14.3 \%$ and $30.7 \% .^{2-6}$ With recent advances in interventional radiology techniques, transcatheter arterial embolization (TAE)

Corresponding author: Hayato Sasaki, MD, Department of Surgery, Institute of Biomedical and Health Sciences, Hiroshima University, 1-2-3 Kasumi, Minami-ku, Hiroshima 734-8551, Japan. 

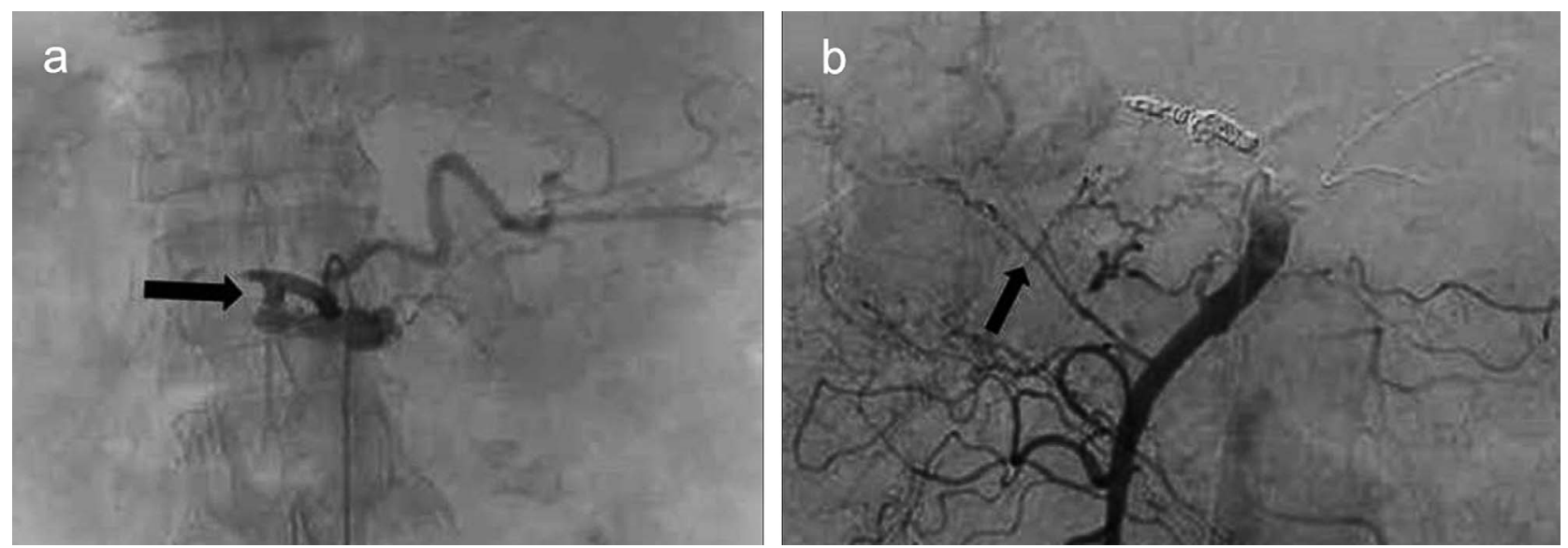

Fig. 1 Celiac arteriography showing extravasation (arrow) from a pseudoaneurysm of the common hepatic artery (a).

Postembolization arteriography showing development of slight collateral flow to the liver from a jejunal branch of the superior mesenteric artery (arrow) (b).

has become an alternative to surgical treatment. $3,5,7,8$ However, it may be difficult to treat these patients with interventional radiology techniques alone, given their often unstable condition. In addition, the inappropriate use of TAE for arterial bleeding, especially after pancreatoduodenectomy, can lead to end-organ infarction and subsequent infection. We report herein our experience with a patient who had bleeding from the superior mesenteric artery (SMA) after pancreatoduodenectomy. This patient was successfully treated using SMA coil embolization followed by creation of an SMA-iliac artery bypass using a greater saphenous vein graft.

\section{Case Report}

A 65-year-old man was diagnosed with borderlineresectable pancreatic adenocarcinoma with tumor abutment of the SMA less than $180^{\circ}$ of the circumference of the vessel wall. He received neoadjuvant radiation therapy (50.4 Gy in 28 fractions) with gemcitabine $\left(1000 \mathrm{mg} / \mathrm{m}^{2}\right.$ on days 1 and 8$)$ plus S-1 $\left(60 \mathrm{mg} / \mathrm{m}^{2}\right.$ on days $\left.1-14\right)$ every 3 weeks for a total of 2 courses. He then underwent pancreatoduodenectomy and pancreaticogastrostomy with portal vein resection, skeletonization of the hepatoduodenal ligament, and dissection of the nerve plexus of the SMA. After surgery, the patient had developed a pancreatic fistula with regional sepsis; therefore, drainage for the pancreatic fistula was continued.

On postoperative day 42 , the patient experienced bleeding from the abdominal drain. Multidetector computed tomography (MDCT) revealed portal vein occlusion and irregular changes in the luminal diameter of the common hepatic artery (CHA), but no pseudoaneurysm or extravasation of contrast were identified. On postoperative day 45 , he developed massive hematemesis with acute hypotension. Emergency angiography revealed active bleeding from a CHA pseudoaneurysm (Fig. 1a). Because the portal vein was occluded, a stent graft was initially placed in an attempt to prevent liver ischemia; however, this proved technically difficult in this hemodynamically unstable patient. We failed to stop the bleeding using the stent graft, and subsequently performed n-butyl-2-cyanoacrylate (NBCA) embolization of the CHA. On the day after embolization, the patient again developed hematemesis. Emergency angiography revealed recanalization of the CHA, which was subsequently reembolized using microcoils. Postembolization angiography demonstrated the development of slight collateral flow to the liver from a jejunal branch of the SMA (Fig. 1b). The patient developed multiple liver abscesses after embolization; these were drained percutaneously using ultrasound guidance.

On postoperative day 64, the patient once again developed sudden, massive hematemesis, with hemodynamic compromise progressing to shock. Emergency angiography revealed active bleeding from the proximal SMA (Fig. 2a). We deemed the use of a stent graft too risky in this emergent situation, because there was little space in the proximal SMA to affix the graft. Therefore, the patient underwent embolization of the SMA using both microcoils and NBCA (Fig. 2b). Immediately after embolization, we attempted to prevent bowel 
Fig. 2 Superior mesenteric arteriography showing extravasation (arrows) from the proximal portion of the vessel (a). Follow-up angiography after coil embolization reveals no extravasation (b).
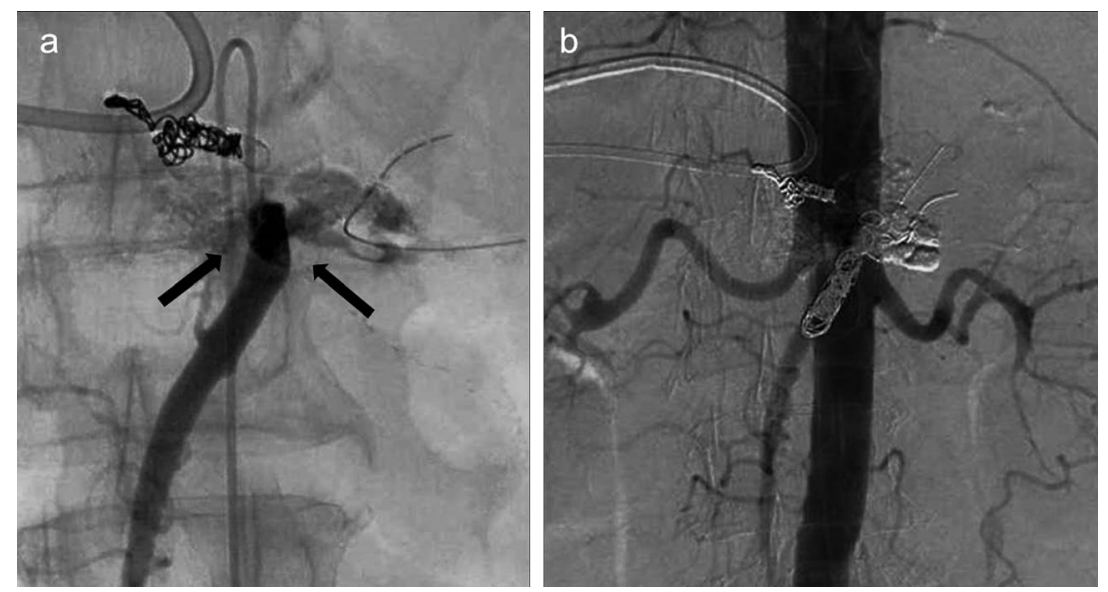

ischemia by creating a bypass graft, using the greater saphenous vein, between the distal SMA and right common iliac artery (Fig. 3). Revascularization of the SMA rapidly improved small-bowel peristalsis, and there was no evidence of ischemia during the operation. The operative time was 299 minutes, and the time between embolization and reperfusion of the SMA was 330 minutes. MDCT on postoperative day 69 confirmed that blood flow to the intra-abdominal organs was from the iliac artery alone via the saphenous-vein graft (Fig. 4). The portal vein remained occluded, as did the CHA and SMA embolization sites. Poor arterial blood flow and occlusion of the portal vein led to worsening of the liver abscesses; however, liver failure did not develop (maximum total bilirubin, $8.9 \mathrm{mg} / \mathrm{dL}$ ). The patient was discharged 122 days after the initial surgery.

\section{Discussion}

$\mathrm{PPH}$ is an uncommon complication, but it can cause significant morbidity. Bleeding from the SMA has an extremely poor prognosis compared with bleeding from the gastroduodenal or hepatic arteries. ${ }^{9}$ The International Study Group of Pancreatic Surgery has classified PPH into 3 grades: A, B, and C. ${ }^{1}$ These grades are determined by taking into account the onset (early, less than 24 hours after the end of the index operation; or late, more than 24 hours later), location (intraluminal or extraluminal), severity (mild or severe), and the clinical impact of the bleeding. In our patient, both bleeding episodes, from the CHA and from the proximal SMA, were classified as $\mathrm{PPH}$ grade $\mathrm{C}$ : life-threatening and requiring immediate treatment.
Open repair of bleeding vessels carries the risk of significant morbidity and mortality in patients with $\mathrm{PPH}$. The less invasive TAE is often used, especially in emergency situations. The success rate of TAE has been reported to be between $63 \%$ and $85 \%$. $3,5,10$ However, using TAE in the hepatic artery after pancreatoduodenectomy carries a high risk of dissection of the hepatoduodenal ligament, including the collateral vessels, potentially causing liver infarction, liver abscess, and necrosis. The incidence of liver abscess after hepatic artery embolization is more than $30 \%$. ${ }^{3,8}$

Recently, stent grafts, which preserve blood flow to prevent end-organ ischemia, have been used for bleeding abdominal vessels. There are several reports of patients with good clinical courses after stent grafting. ${ }^{11-13}$ However, the placement of a stent

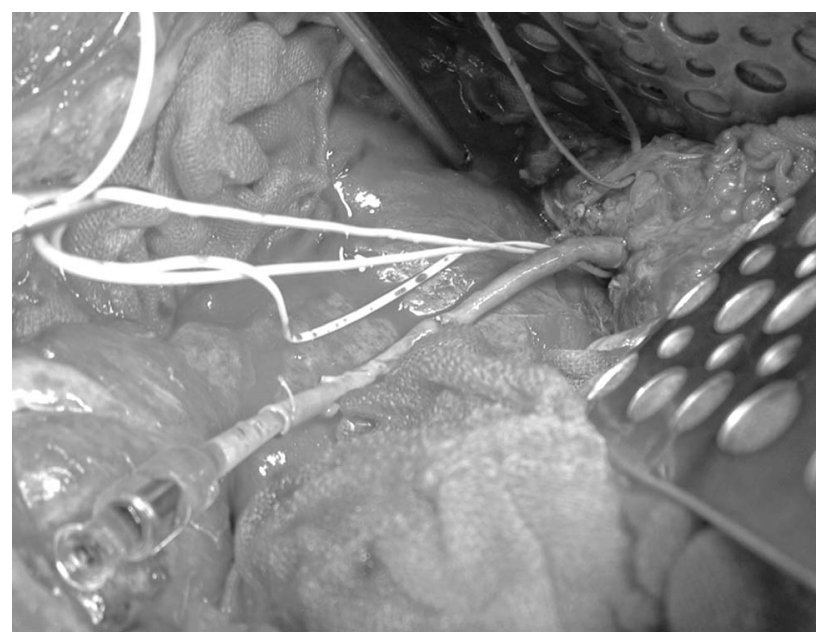

Fig. 3 Bypass is created between the distal superior mesenteric artery and the right common iliac artery, using a greater saphenous vein graft. 


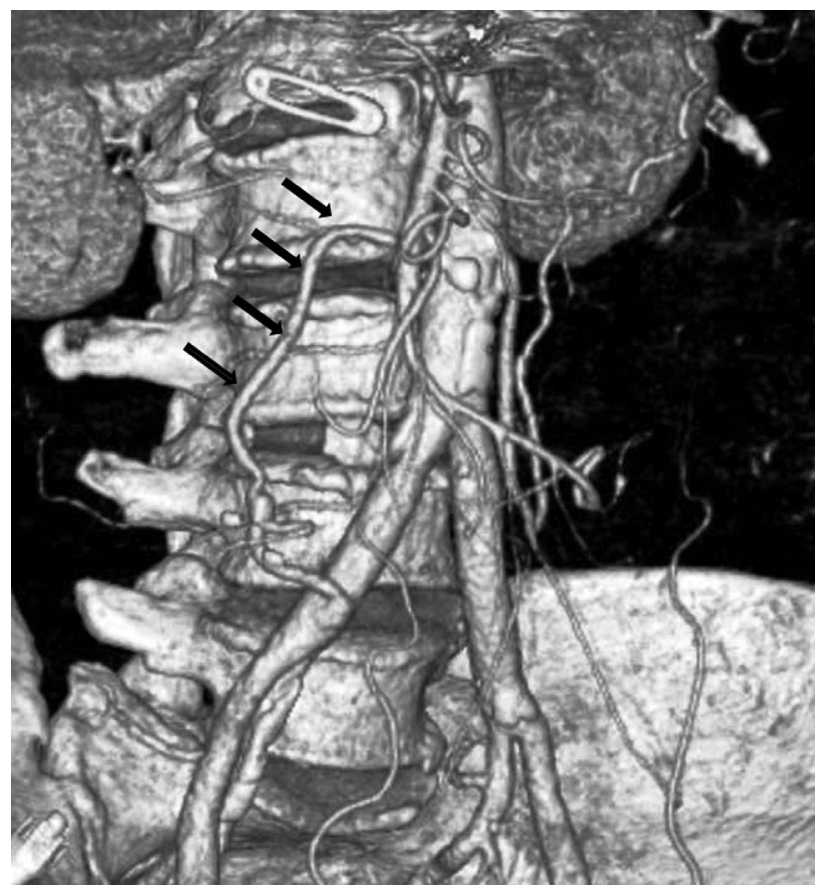

Fig. 4 Three-dimensional CT angiography showing intraabdominal blood flow only from the iliac artery via the saphenous vein graft (arrows).

graft may be technically challenging, and the success of the procedure depends on the technique used and the experience of the radiologists or cardiologists involved, as well as the availability of the devices, particularly in emergent situations. Indeed, we made an unsuccessful attempt to deploy a CHA stent graft but were stymied by the technical difficulty in our hemodynamically unstable patient. Furthermore, there is always the risk of stent-graft infection, particularly in patients with preexisting infection in the region. Suzuki et al ${ }^{14}$ reported a patient with intraperitoneal bleeding from the SMA after pancreatoduodenectomy, treated with a stent graft. The patient incurred a stent-graft infection and died of sepsis 5 months later. Although stent grafts have benefits over TAE, long-term safety data are needed.

While the use of stent grafts for bleeding from the SMA is well documented, there are few reports of successful treatment with a combined endovascular and surgical approach. Saito et $a l^{15}$ reported a patient who had delayed massive hemorrhage from a pseudoaneurysm of the SMA after distal pancreatectomy. This patient was treated using coil embolization and bypass grafting between the SMA and the abdominal aorta, using a saphenous vein graft. In our patient, there was a high possibility of bowel ischemia if TAE only was used at the proximal SMA. In addition, based on the previous failure of stent grafting in the $\mathrm{CHA}$, we anticipated technical difficulties. Therefore, we performed SMA embolization first, to provide hemodynamic stability, then immediately created the SMA-iliac artery bypass. To the best of our knowledge, this is the first patient with SMA bleeding after pancreatoduodenectomy to be successfully treated using this hybrid approach.

Two bypass routes can be usually used to graft the abdominal aorta or the common iliac artery as the origin of mesenteric revascularization for treatment of intestinal ischemia. ${ }^{16} \mathrm{SMA}-\mathrm{iliac}$ artery route is relatively long and retrograde bypass grafting; however, it has several benefits that are less invasive and technically simple compared with SMA-aorta bypass grafting, because side or total clamping of the aorta is not necessary. Furthermore, it was expected that our patient had strong adhesions caused by postoperative pancreatic fistula and subsequent hemorrhage in the upper abdomen. Therefore, we chose the common iliac artery for the origin of the bypass graft to avoid the adhesions in this postoperative and emergent situation.

In conclusion, the combination of SMA embolization and SMA-iliac artery bypass grafting is a viable option for treating postoperative SMA bleeding in hemodynamically unstable patients.

\section{References}

1. Wente MN, Veit JA, Bassi C, Dervenis C, Fingerhut A, Gouma $\mathrm{DJ}$ et al. Postpancreatectomy hemorrhage (PPH): an International Study Group of Pancreatic Surgery (ISGPS) definition. Surgery 2007;142(1):20-25

2. Rumstadt B, Schwab M, Korth P, Samman M, Trede M. Hemorrhage after pancreatoduodenectomy. Ann Surg 1998; 227(2):236-241

3. Sato N, Yamaguchi K, Shimizu S, Morisaki T, Yokohata K, Chijiiwa K. Coil embolization of bleeding visceral pseudoaneurysms following pancreatectomy: the importance of early angiography. Arch Surg 1998;133(10):1099-1102

4. Yekebas EF, Wolfram L, Cataldegirmen G, Habermann CR, Bogoevski D, Koenig AM et al. Postpancreatectomy hemorrhage: diagnosis and treatment: an analysis in 1669 consecutive pancreatic resections. Ann Surg 2007;246(2):269-280

5. Limongelli $\mathrm{P}$, Khorsandi SE, Pai M, Jackson JE, Tait P, Tierris J et al. Management of delayed postoperative hemorrhage after pancreaticoduodenectomy: a meta-analysis. Arch Surg 2008; 143(10):1001-1007

6. Eckardt AJ, Klein F, Adler A, Veltzke-Schlieker W, Warnick P, Bahra $\mathrm{M}$ et al. Management and outcomes of haemorrhage 
after pancreatogastrostomy versus pancreatojejunostomy. $\mathrm{Br} \mathrm{J}$ Surg 2011;98(11):1599-1607

7. de Castro SM, Kuhlmann KF, Busch OR, van Delden OM, Laméris JS, van Gulik TM et al. Delayed massive hemorrhage after pancreatic and biliary surgery: embolization or surgery? Ann Surg 2005;241(1):85-91

8. Tani M, Kawai M, Yamaue H. Intraabdominal hemorrhage after a pancreatectomy. J Hepatobiliary Pancreat Surg 2008;15(3): 257-261

9. Miura F, Asano T, Amano H, Yoshida M, Toyota N, Wada K et al. Management of postoperative arterial hemorrhage after pancreato-biliary surgery according to the site of bleeding: relaparotomy or interventional radiology. J Hepatobiliary Pancreat Surg 2009;16(1):56-63

10. Okuno A, Miyazaki M, Ito H, Ambiru S, Yoshidome $H$, Shimizu $\mathrm{H}$ et al. Nonsurgical management of ruptured pseudoaneurysm in patients with hepatobiliary pancreatic diseases. Am J Gastroenterol 2001;96(4):1067-1071

11. Shrikhande GV, Khan SZ, Gallagher K, Morrissey NJ. Endovascular management of superior mesenteric artery pseudoaneurysm. J Vasc Surg 2011;53(1):209-211
12. Wallace MJ, Choi E, McRae S, Madoff DC, Ahrar K, Pisters P. Superior mesenteric artery pseudoaneurysm following pancreaticoduodenectomy: management by endovascular stentgraft placement and transluminal thrombin injection. Cardiovasc Intervent Radiol 2007;30(3):518-522

13. Nakai M, Sato H, Sato M, Ikoma A, Minamiguchi H, Kawai N et al. Successful endovascular treatment of iliac arteriovesical fistula with secondary stent-graft infection. J Vasc Interv Radiol 2013;24(9):1409-1412

14. Suzuki K, Mori Y, Komada T, Matsushima M, Ota T, Naganawa S. Stent-graft treatment for bleeding superior mesenteric artery pseudoaneurysm after pancreaticoduodenectomy. Cardiovasc Intervent Radiol 2009;32(4):762-726

15. Saito T, Tsuchiya T, Kenjo A, Kimura T, Sato Y, Saito T et al. Successful treatment of pseudoaneurysms of celiac and superior mesenteric arteries by combined endovascular and surgical approach. J Hepatobiliary Pancreat Surg 2008;15(4):444448

16. Foley MI, Moneta GL, Abou-Zamzam AM Jr, Edwards JM, Taylor LM Jr, Yeager RA et al. Revascularization of the superior mesenteric artery alone for treatment of intestinal ischemia. $J$ Vasc Surg 2000;32(1):37-47 